\title{
CONSIDERACIONES TEÓRICAS Y EMPÍRICAS ACERCA DEL RESALE PRICE MAINTENANCE ${ }^{*}$
}

\author{
DAVID ANDRÉS CAMARGO MAYORGA*", JOHAN RICARDO PÁEZ CASTILLO \\ \& ÁNGEL DAVID RONCANCIO GARCÍA"*n \\ PONTIFICIA UNIVERSIDAD JAVERIANA, UNIVERSIDAD MILITAR NUEVA GRANADA
}

Recibido/ Received/Recebido: 23/08/2010 - Aceptado/ Accepted/Aprovado: 27/10/2010

\begin{abstract}
Resumen
Este documento, explora algunas de las consideraciones teóricas y empíricas más importantes acerca del Resale Price Maintenance (RPM) el cual se ha convertido no solo en un problema de orden económico, sino legal. Para ello, el tema se abordará en dos aspectos. En primer lugar, se describen las implicaciones teóricas del RPM, y por otra parte, se muestra la evidencia empírica encontrada a partir de una exploración documental. De manera que la reflexión planteada sugiere que las restricciones verticales evitan el free riding tanto en fabricación como en distribución, dada la tentación de aprovechar el esfuerzo de otros comercializadores en gastos de publicidad, entrenamiento de asesores, reputación de la marca, inversiones en salas de exhibición, etc., en beneficio propio, además de reducir la incertidumbre.
\end{abstract}

Palabras clave: Fijación de precios de reventa, Restricciones verticales, Free riding, y Margen monopólico.

\section{THEORETICAL AND EMPIRICAL CONSIDERATIONS ABOUT RESALE PRICE MAINTENANCE}

\begin{abstract}
This document explores some of the most important theoretical and empirical considerations about Resale Price Maintenance (RPM), which has become not only an economic but also a legal problem. In order to achieve that, the topic will be reviewed in two aspects. First, description of theoretical implications of RPM, and on the other hand, empirical evidence found since a documental exploration. Thus, the reflection stated suggest that vertical restrictions avoid free riding in both elaboration and distribution, given the temptation to take advantage of other traders' effort in advertising expenses, advisers' training, benchmarking reputation, investments in exhibition rooms, etc., for own benefit besides reducing uncertainty.
\end{abstract}

Key words: Resale price maintenance, Vertical restraint, Free riding, monopolistic margin.

\footnotetext{
Artículo de reflexión producto del curso de Organización Industrial de la Maestría en Economía de la Pontificia Universidad Javeriana.

* Economista y estudiante de la Maestría en Economía de la Pontificia Universidad Javeriana. Docente hora cátedra de la Facultad de Ciencia Económicas y asistente de investigación del Grupo de Estudios Contables (GECONTA) - Universidad Militar Nueva Granada. Correo electrónico: david.camargo@unimilitar.edu.co

*** Economista y estudiante de la Maestría en Economía de la Pontificia Universidad Javeriana. Correo electrónico: jricardopaez@ yahoo.com

**** Contador Público Universidad Militar Nueva Granada, Filósofo y estudiante de la Maestría en Filosofía de la Pontificia Universidad Javeriana. Docente investigador de la Facultad de Ciencia Económicas y miembro de GECONTA - Universidad Militar Nueva Granada; Correo electrónico: angel.roncancio@unimilitar.edu.co
} 


\title{
CONSIDERAÇÕESTEÓRICAS E EMPÍRICAS SOBRE O RESALE PRICE MAINTENANCE
}

\begin{abstract}
Resumo
Nesteartigo exploram-se algumas considerações teóricas e empíricas importantes sobre o Resale Price Maintenance (RPM), que se tornou um problema financeiro e legal. Primeiro, se descrevem as implicações teóricas do RPM; logo, se indica a evidência empírica encontrada mediante pesquisa documental. Assim, o tema proposto sugere que as restrições verticais impedem o free riding na manufatura e na distribuição, porque se tende à sacar proveito do esforço de outros comerciantes em custos de publicidade, treinamento de assessores, aceitação da marca, investimentos em exibição, etc., para seu próprio benefício. Além disso, é reduzida a incerteza.

Palavras-chave: Fixação de preços de revenda, restrições verticais, free riding, margem monopólico.

Camargo, D., Páez, J. \& Roncancio, A. (2010) Consideraciones teóricas y empíricas acerca del resale price maintenance. En: Revista de la Facultad de Ciencias Económicas de la Universidad Militar Nueva Granada. rev.fac.cienc.econ, XVIII (2)
\end{abstract}

JEL: D2, L5.

\section{Introducción}

Las restricciones verticales se definen como prácticas o acuerdos convenidos entre dos o más empresas, en donde cada firma actúa en fases económicas diferentes. Se presenta como alternativa a la integración vertical cuando ésta no es conveniente, ni posible. En consecuencia, los acuerdos regulan las condiciones en las que las partes compran, venden o revenden determinados bienes o servicios (Martínez \& Quintana, 2005).

Las motivaciones que llevan a la imposición de restricciones verticales y su incidencia sobre el bienestar han sido altamente debatidas en los círculos académicos. Por un lado, están quienes consideran que este tipo de acuerdos hacen más eficiente la estructura vertical y que las autoridades no deben restringirlas; pero alternativamente, están quienes sugieren que cualquier prohibición a comerciar libremente por una de las partes, es perjudicial y contrario al bienestar.

Las restricciones verticales adquieren diferentes formas, dentro de las cuales se encuentran los precios no lineales, las cantidades obligatorias, los requerimientos de servicio, la negativa a suministrar, la distribución exclusiva en beneficio de un único distribuidor, la distribución restringida a bienes de un sólo productor, las ventas atadas y el resale price maintenance (RPM), traducido al español como fijación o mantenimiento de precios de reventa.

Este último, es un mecanismo en el cual el precio de reventa no lo establece el distribuidor, sino que lo fija el fabricante o mayorista, lo que quiere decir que los precios de los bienes o servicios vienen preestablecidos, y los distribuidores no pueden vender ni por debajo, ni por encima de lo pactado. Lo que es más, el RPM es tratado en la literatura como un problema tanto económico como legal (Curtis, 1938), y es desde estos dos enfoques de análisis, de donde se han orientado las investigaciones sobre el tema.

El RPM se conviene a través de contratos, donde los fabricantes se aseguran que el comercializador minorista no pueda influir los precios, que en otras condiciones hallaría óptimos, pero que en este escenario se deben ceñir a lo pactado. 
En síntesis, el presente artículo tiene como propósito hacer una exploración del RPM principalmente en el ámbito económico, y para tal efecto se divide en tres partes: la primera recorre algunas investigaciones acerca de las implicaciones teóricas del RPM, la segunda muestra la evidencia empírica encontrada a partir de la revisión documental, y la tercera presenta las conclusiones.

\section{Investigaciones acerca de las implicaciones teóricas del RPM}

El RPM tiene implicaciones de carácter teórico como lo muestra la literatura sobre el tema. Nuestro interés no es dar cuenta de las teorías en general sobre este tipo de restricción vertical, sino abordar las principales implicaciones teóricas de su aplicación.

En primer lugar, Rey \& Vergé (2008) definen el RPM como un acuerdo en el cual el precio que paga el consumidor no es fijado por el distribuidor, sino por el productor. En el mismo sentido, Elzinga \& Mills (2006) lo precisan diciendo que:

"Son contratos en los que el fabricante $y$ el distribuidor acuerdan un precio mínimo o máximo al cual el minorista le venderá a los compradores".

Elzinga \& Mills exponen además que dentro de las razones por las cuales los fabricantes imponen estos precios, están que los consumidores perciben diferente el producto que compran a partir del servicio que reciben y lo que pagan, de lo cual surgen fallas de mercado. Además, enfatizan en la conveniencia del RPM por la violación de dos supuestos microeconómicos: información perfecta y homogeneidad del producto. Por tanto, la información asimétrica en el mercado minorista y el hecho que la demanda por un producto pueda depender tanto del precio como de la calidad del servicio prestado por parte del distribuidor, son la evidencia de dichas fallas.

Aun más, el RPM puede impedir el Free riding (traducido al español usualmente como "el problema del polizón”), dado que algunos vendedores no se esfuerzan tanto como otros para lograr cierto número de ventas, sino que esperan a que estos otros se esfuercen por vender más. Para entender mejor esta situación, supóngase una persona que visita una tienda donde le muestran detalladamente un producto, pero no hace la compra por el elevado precio del artículo, en cambio va a otro almacén donde le resulta más barato, dado que allí no se invierte en servicio de asesoría comercial.

De lo anterior se deduce que muchos productores usan la estrategia del RPM para disminuir la competencia en los mercados en que operan, planteando que en ausencia de esta restricción vertical, los consumidores van a comprar donde simplemente les ofrecen menores precios sin importar el servicio que el minorista pueda prestarles (Telser, 1960).

En concordancia, Chen \& Chen (2005) realizan un análisis respecto a las razones por las cuales se utiliza este tipo de restricción. La primera y más importante se la atribuyen al problema del free rider en la distribución, es decir, en los servicios de venta. De modo que los distribuidores no verán conveniente invertir en mejorar su servicio, dado que esto no se verá reflejado en los beneficios por la venta de sus productos, a menos que sea en un bien que requiera algún tipo de demostración. Chen (1999) sugiere que el RPM se establece para permitir a los distribuidores capturar la demanda por medio del servicio, de ahí la intención de los vendedores de invertir en este valor agregado.

Asimismo, Chen incluye el concepto de incertidumbre, al afirmar que el RPM la reduce, aunque sugiere que su implementación genera costos de transacción, tales como mecanismos de monitoreo e inversiones que convencen o hacen desistir a los minoristas de entrar en el mercado bajo dicha restricción vertical. En efecto, los costos alusivos al RPM no pueden ser cero, lo que en caso contrario se considera un supuesto poco realista, por lo cual el RPM minimiza el riesgo, aunque no debe ser entendido como una herramienta para concentrar poder de mercado (Chen \& Chen, 2005), aun cuando los distribuidores llegarían a tener mucha competencia entre ellos de no existir dicha fijación de precios (Chen, 1999).

Por otro lado, Grether (1935) plantea que los métodos de fijación de precios solo podrían ser efectivos 
para un número muy pequeño de bienes de consumo, como por ejemplo los que tienen dificultades en su distribución por la gran cantidad de intermediarios en el proceso, además de los que son marcas registradas (trademark) y los que tienen que ver con conductas humanas, tales como el liderazgo. No obstante, Grether cuestiona la efectividad del control de precios en varios aspectos, principalmente argumenta que los minoristas pueden encontrar mecanismos para modificarlos e incurrir en gastos adicionales de mercadeo que pueden desvanecer el margen de beneficios que en teoría obtendrían.

En el caso de las implicaciones en torno al bienestar, Wang (2009) realiza un análisis en una situación en donde hay distribuidores heterogéneos. Escenario en el cual un fabricante opta por vender sus productos en dos regiones distintas, con costos idénticos para los minoristas dentro de una región, pero distintos en otros lugares. De donde concluye que, la política de RPM es perjudicial para el consumidor, ya que aumenta los costos de los distribuidores, es decir, los que se transfieren vía precios al consumidor final. Por ejemplo, Wang describe el caso de las farmacias británicas diciendo que si se:

"...Prohíben las políticas de RPM muchas droguerías irían a la quiebra, porque ellos dependen mucho del mercado de mostrador [OTC en inglés], y no estarían en capacidad de competir con estrategias agresivas de precios y tácticas promocionales como las que se hacen en los supermercados" $(2009,4)$.

\section{Evidencia empírica acerca del RPM}

Con respecto a la evidencia empírica, es de aclarar que se entiende por tal, la comprobación, medición y verificación en la realidad de la implementación del RPM, así tenemos por un lado resultados operativos y mediciones económicas, y por ultimo algunos estudios de caso que mencionan el resultado de aplicar el RPM. En este último aspecto, es de señalar que los casos estudiados combinan la problemática legal con la económica.
Deneckere, Marvel \& Peck (1997) prueban la adopción del RPM en un mercado competitivo, mediante un modelo donde existe un fabricante monopólico y un mercado competitivo en la distribución. Encontrando que se obtienen mayores beneficios implantando el RPM, que en el escenario de libre competencia, incluso para el consumidor y el excedente que éste captura. Con el mismo propósito Gilligan (1986) analiza los efectos competitivos de las políticas de RPM, partiendo de la ambigüedad de los resultados obtenidos en su aplicación. Insistiendo en que la estructura de las empresas fabricantes y de la industria como tal, mantiene una firme incidencia en los efectos de estas medidas.

En contravía de lo anteriormente expuesto, Gilligan nombra algunas características generales de esta medida, y como que puede ir en contra de la competencia debido a que permite la "cartelización" en la cadena de producción, o discriminación de precios por parte de un productor monopolista; sin dejar de lado las ventajas que trae consigo, siendo una de éstas la reducción del riesgo moral en la distribución. Gilligan basa su estudio en el desarrollo de una metodología empírica para la estimación de los efectos asignativos y productivos de una política de RPM, arrojando como resultado que este tipo de restricciones verticales son pieza clave al determinar los beneficios o ganancias de las empresas.

Como tal el RPM también se puede analizar desde la parte legal, sin perder de vista las connotaciones económicas que este tipo de decisiones trae consigo. En este sentido, la fijación de precios se evidencio en los Estados Unidos como una manera de infringir la Ley Sherman, promulgada en 1890 para conseguir preservar la libre competencia. De dicha Ley se rescatan por su importancia los siguientes dos parágrafos:

"...Se declara ilegal todo contrato, combinación en forma de "trust" (concentración de empresas) o de otra clase, o conspiración restrictiva del tráfico o del comercio entre los estados o con países extranjeros... [Se considera infractor a la ley]... a toda persona que monopolice o trate de monopolizar cualquier parte 
de la actividad o el comercio entre o los diversos estados o con naciones extranjeras y toda persona que se coaligue o entre en combinación con cualquier otra persona o personas para monopolizar cualquier parte del comercio entre los estados federados o con otros países". ${ }^{1}$

Varios análisis se han hecho de casos ocurridos en la época cuando recién salía esta disposición, por ejemplo Sheffet \& Scammon (1985) hacen un resumen de los más importantes, destacando el caso de "Dr. Miles Chemical" en 1911, cuando esta compañía argumentó que el minorista "Park \& sons Co" estaba vendiendo sus productos con precios más bajos que los estipulados por el fabricante. La corte $e^{2}$ en su momento estipuló que la actitud del fabricante respecto al minorista era algo similar a si los minoristas hubiesen pactado los precios, lo que en resumen era catalogado como una actitud anticompetitiva.

El más mínimo uso del RPM como restricción vertical fue una falta per sé a partir de aquel caso de "Dr. Miles Chemical", hasta el año 2007 cuando se reversó esta consideración legal en el marco del caso que enfrentaba las compañías de cueros "Leegin" y "PSKS Inc.".

La corte al pronunciarse en contra de la ilegalidad per sé, argumento que los casos se deben evaluar con la "regla de la razón", lo que quiere decir que cada caso se debe analizar por separado y sopesando los argumentos que cada empresa tiene para demostrar su conducta, evaluando los aspectos competitivos y anticompetitivos. Además, la corte esgrimió que se debe diferenciar entre las actitudes anticompetitivas que pueden resultar peligrosas para los consumidores, y las restricciones que pueden llevar a incrementar la competitividad dentro de un mercado (Antitrust Institute, 2009). La corte, al emitir ese fallo se pronunció respecto al hecho que los futuros análisis de casos concernientes a RPM deben incluir "información específica", la intención de la restricción (si es para concentrar poder de mercado) y sus respectivos efectos.

El caso de "Leegin" fue analizado por Leary \& Mintzer (2008), quienes mencionan que esta compañía era una pequeña fabricante de productos de cuero que enfocaba sus estrategias en boutiques, en las que se valoraba mucho el servicio al cliente. Sus políticas respecto a RPM tenían que ver con acuerdos firmados con sus distribuidores, mientras que su mercado era competitivo tanto a nivel de fabricante como a nivel de distribución. Si bien en el juicio se encontró que hubo acuerdos de "Leegin" con sus distribuidores, era muy difícil que se comprobara una conducta anticompetitiva.

En el mismo sentido, Sheffet \& Scammon (1985) citan otros casos históricos, como el de "Colgate" en 1919 en donde este fabricante se negaba a negociar con quienes no aceptaran sus precios de reventa, caso en el cual la corte falló a favor del fabricante al manifestar que era libre de negociar con quien quisiera. Asimismo, fue el caso de "Parke Davis" en 1960, que consistió en que esta compañía no negociaba con los minoristas que cobraban al consumidor por debajo del precio de reventa fijado por el fabricante, e incluso prohibía a los mayoristas que tuvieran nexos con dichas tiendas. Con la misma estrategia "Monsanto" en 1984 hizo desaparecer a "Spray Rite". En este último caso la corte determinó que no había suficiente evidencia para demostrar que "Monsanto" había hecho adrede algo anti-reglamentario para acabar con el minorista.

En 1987 se expide la Ley S.430 que establecía que para que hubiera conducta ilegal, era necesario demostrar que se presentaba una situación de alianza entre el fabricante y varios minoristas, sugiriendo cómo se deberían relacionar distribuidores y productores. Así, cuando existieran cadenas de distribución establecidas, planteaba que se debía actuar unilateralmente evitando la coerción, y cuando se

\footnotetext{
1 Tomado del enlace de la Universidad de Málaga: http://www.eumed.net/libros/2008a/369/Sherman\%20Act\%20de\%201890. htm

2 La Corte a la que haremos referencia de aquí en adelante es la Supreme Court of the United States.
} 
tratara de nuevos distribuidores, pedía que se buscara la integración vertical, que se anunciasen con claridad las políticas de RPM y que se hallara la manera de evitar negociaciones entre minoristas (Greco, 1992).

Como hemos visto, los análisis anteriores han tocado el ámbito de lo legal en cuanto a la aplicación del RPM en algunos sectores y empresas. Pero es importante mencionar que el estudio de caso no ha dejado de lado este aspecto como lo muestra Grether, Yamey y Munro Lee.

En el caso de Grether (1935) se enfatiza en casos específicos ocurridos después de la sanción de la "Ley de comercio justo" (Fair trade Act) de 1931, en la que los contratos de RPM establecían precios mínimos y escasos márgenes de ganancia para los minoristas, lo que desincentivo a estos últimos para invertir en publicidad o en un mejor servicio.

Yamey (1950) analiza la reacción de las compañías minoristas y las formas para recortar el precio dentro del contexto de RPM, afirmando que dicho recorte de precios puede ser tanto deseable como indeseable; siendo deseable cuando se toma ventaja de la elasticidad de la demanda o de una reducción de costos, mientras que es nocivo cuando la disminución de precios se presenta en un entorno para destruir a los competidores.

Además, Yamey (1950) describe los diversos tipos de contratos que se usan dentro de RPM. El primero que menciona, enfatiza en la situación en que el fabricante unilateralmente impone sus condiciones para la venta de sus productos; en el segundo tipo, resalta que el fabricante se encuentra bajo presión de un gremio de distribuidores lo suficientemente hábil como para realizar un boicot; y como tercer tipo, describe aquel en el que el proceso de fijación de precios se realiza mediante una colaboración entre agremiaciones de fabricantes y minoristas.

Algo interesante que muestra Yamey (1950) es que para cierto tipo de bienes con elasticidad infinita (o que él llama "comprados por impulso"), la mejor medida de promoción es el RPM, así que en su momento este fue un argumento para no prohibir esta restricción vertical, debido a que la demanda caería; explicando que si hay un precio efectivo y con un margen interesante para los minoristas, estos últimos se esforzarían para prestar un mejor servicio y promocionar el producto de forma adecuada.

Para Munro Lee (1959) el interés está en mencionar las empresas donde se ha utilizado RPM como General Electric, Parker y Kodak. Presenta además los resultados de una encuesta donde se concluyó que los fabricantes no estaban realizando una buena labor con el RPM, puesto que estaban incurriendo en diversos problemas, tales como el aumento de costos y decisiones adversas en las cortes, lo que se resumía en no utilizar el RPM como restricción vertical.

Muy posteriormente, Romano (1994) analiza el problema del riesgo moral y del doble riesgo moral, describiendo cuándo ocurre desde la empresa fabricante (la empresa hace movimientos que influencian la demanda sin modificar los precios), y desde la firma que comercializa el producto (costos de publicidad, mejor servicio).

Dentro de sus hallazgos y conclusiones, Romano (1994) plantea que los contratos funcionan sólo cuando hay riesgo moral de la empresa que comercializa el producto, y fallan cuando existe el doble riesgo, añadiendo que los contratos óptimos generalmente conllevan el uso de precios de reventa. De este modo indica que con precios máximos o mínimos de reventa se puede llegar a óptimos. Ahora bien, el riesgo moral se puede detener según lo plantea Romano, con la fijación de los precios finales para los minoristas.

En Europa, la utilización de RPM en algunos mercados ha llevado a fuertes cuestionamientos a estas conductas, aunque la OCDE (1997) argumenta que esta es una buena forma de acabar con el doble margen monopólico. Práctica que se refiere a la existencia de dos empresas, una que es monopólica en la fabricación y la otra que tiene el monopolio en la distribución, lo que redunda en pérdidas de eficiencia, en caídas en las ganancias de las compañías, y en precios más altos para el consumidor. Este doble margen depende de la elasticidad de la demanda que enfrenta cada monopolio. 
La OCDE enfocándose en el "free riding" y en la práctica denominada "remover la crema" -la cual hace alusión a las tiendas y página de internet que se especializan en vender libros más baratos- concluye que la fijación de precios de reventa es un obstáculo para la competencia, debido a que impide nuevas y más eficientes formas de distribución de los productos. Dice también que el RPM lleva a conductas colusivas y a la formación de carteles, las cuales redundan en la disminución de la competencia entre los distribuidores.

Rey \& Vergé (2004) analizan el caso en que los fabricantes y los distribuidores tienen una relación entrelazada, asumiendo para su análisis dos fabricantes y dos distribuidores. En esta situación cada uno vende un producto de cada fabricante; llegando a la conclusión que bajo RPM, y con cada fabricante fijando el precio, los distribuidores no tendrán ganancias y los precios serán monopólicos. Su análisis cambia cuando surge competencia imperfecta entre los distribuidores, donde si bien los precios pueden seguir siendo cercanos al nivel de monopolio, ahora la fijación de estos depende de un equilibrio donde los distribuidores buscan precios altos (para aumentar su ganancia) y los fabricantes buscan precios bajos (para que las ganancias de los distribuidores no sean tan altas).

En Europa también se discute desde el punto de vista legal la utilización de RPM, Gogeshvili (2002) esgrime argumentos tanto a favor como en contra de estas políticas. A favor del RPM mínimo, dice que ayuda a disminuir el free riding, anima la entrada de algunas empresas para operar en los mercados, y promueve la provisión de servicios. En contra, dice que puede llegar a ser una actitud anticompetitiva $y$ que puede llevar a incrementos injustificados en los precios de algunos bienes. Como conclusión, este autor invita a que en Europa se remuevan los castigos per sé y más bien se autorice a usar el RPM cuando este conduzca a promover la competencia.

Finalmente, Piccolo \& Martimor (2003) analizan el RPM con información asimétrica. En su investigación, ellos comparan el RPM y los arreglos de cantidades fijas en un contexto de monopolios sucesivos con selección adversa y riesgo moral. Los autores argumentan que es más flexible cuando se hacen arreglos de cantidades fijas en el sentido que si bien el fabricante establece las cantidades, los minoristas están en libertad de elegir si invierten en mejorar su servicio o en cualquier estrategia que les ayude a vender más. Bajo información asimétrica se presenta un doble margen monopólico que lleva al fabricante a vender por encima del costo marginal. Ellos concluyen que las cantidades con RPM serán menores que las óptimas cuando existe información completa.

\section{Conclusiones}

En términos generales, las investigaciones sugieren que las restricciones verticales pueden solucionar diversos problemas dentro de los que se incluye el doble margen monopólico por la presencia de monopolios sucesivos en fabricación y distribución; Sin embargo, no constituye la única solución a la interacción de los agentes (productor, distribuidor minorista y consumidor final). A favor de la aplicación del RPM, según la evidencia empírica mencionada, ayuda a evitar el free riding tanto en fabricación como en distribución, dada la enorme tentación de aprovechar el esfuerzo de otros comercializadores en términos de gasto en publicidad, entrenamiento de asesores, reputación de la marca, inversiones en salas de exhibición, etc., en beneficio propio. En sintonía con Chen (1999), quien presenta una mirada hacia el "por qué" del RPM, e inicia preguntándose:

"Qué razón tendría un fabricante para limitar los precios a los que venden los minoristas sus productos".

Se puede concluir de sus respuestas y de la bibliografía revisada, que el RPM es solución al problema del Free riding por parte de los minoristas y explicaría el por qué mientras unos de ellos exhiben el producto suntuosamente y lo promocionan (lo cual incrementa sus costos), otros venden y obtienen las mismas ganancias sin invertir un sólo centavo en su promoción. Asimismo, el RPM es útil para corregir el hecho de que los minoristas puedan acordar discriminación de precios en situaciones en las que cobran un precio a unos clientes y otro precio muy 
distinto a otros, $\mathrm{u}$ ofreciendo descuentos a cierto tipo de consumidores.

\section{Referencias}

Antitrust Institute (2009). "Antitrust Analysis of resale Price maintenance after Leegin". En: Special issue of Antitrust bulletin. Disponible en: http://www. antitrustinstitute.org/archives/files/ Call\%20for\%20papers\%20-\%20Antitrust\%20Analysis\%20 of\%20RPM\%20after\%20Leegin\%20_2_120420081709.pdf.

Curtis, A (1938). "Resale price maintenance". En: Revue canadienne d'Economique et de Science politique, 4 (3): 350361.

Chen, Y (1999). "Oligopoly Price Discrimination and Resale Price Maintenance". En: The RAND Journal of Economics, 30 (3): 441-455.

Chen, G \& Chen, M (2005). "Incentive for resale price maintenance from a risk perspective: using a real options analysis". En: Fair Trade Quaterly, 14 (1): 135-151.

Deneckere, R. Marvel, H \& Peck, J (1997). "Demand uncentainty and price maintenance: Markdowns as destructive competition". En: The American Economic review, 87 (4): 619 - 641.

Elzinga, K \& Mills, D (2006). "The Economics of resale Price maintenance". En: Collins, W. (ed). Competition law and policy. American Bar Association, Forthcoming. Disponible en: http://ssrn.com/abstract $=926072$

Gilligan, T (1986). "The Competitive Effects of Resale Price Maintenance". En: The RAND Journal of Economics, 17 (4): 544556.

Gogeshvili, M (2002). "Resale Price maintenance: A dilemma in EU Competition Law". En: The Georgian Law Review, Vol. 5, No. 2, 2-3.

Greco, A (1992). "The Resale Price Maintenance Struggle: Its Legislative Updating". En: American Journal of Economics and Sociology, 51 (2): 173-186.
Grether, E (1935). "Resale Price Maintenance and the Consumer". En: American Marketing Journal, 2 (3): 144-149.

Leary, T \& Mintzer, E (2008). "The future of Resale Price Maintenance now that Dr. Miles is dead". En: NYU Journal of law and business, Vol. 4-(303), 303 -342, disponible en : http:// www.ftc.gov/opp/workshops/rpm/may09/docs/tleary.pdf

Martinez, M \& Quintana, E (2005). "Contratos de exclusividad y ventas atadas: cuando lo atado es la exclusividad". Boletín Latinoamericano de Competencia, Unión Europea.

Munro Lee, S (1959). "Problems of Resale Price Maintenance". En: The Journal of Marketing, 23, (3): 274-281.

OCDE (1997). "Resale price maintenance". Disponible en: http:// www.oecd.org/dataoecd/35/7/1920261.pdf

Piccolo, S \& Martimor, D (2003). "Resale price maintenance under asymetric information". En: Centre for estudies of economics and finance. Working paper \# 107.

Rey, P \& Vergé, T (2008). "Economics of vertical restraints". En: Buccirossi, P. (ed) Handbook of Antritrust economics. London: The MIT Press.

Rey, P \& Vergé, T (2004). "Resale Price Maintenance and Horizontal cartel". En: CMPO Working Paper Series No. 02/047.

Romano, R (1994). "Double Moral Hazard and Resale Price Maintenance". En: The RAND Journal of Economics, 25, (3): 455-466.

Sheffet, M \& Scammon, D (1985). "Resale Price Maintenance: Is It Safe to Suggest Retail Prices?" En: The Journal of Marketing, 49: (4): 82-91.

Telser, L (1960). "Why should manufacturers want fair trade?" Journal of law and economics. 3: $86-105$.

Yamey, B (1950). "Notes on Resale Price Maintenance". En: Económica, New Series, 17 (67): 254-265

Wang, H. (Recuperado el 25 de mayo de 2009) "Retail heterogeneity and Resale Price maintenance". Disponible en: http:// www.cenet.org.cn/cn/CEAC/\%E7\%AC\%AC\%E4\%B8\%89\% E5\%B1\%8A\%E5\%85\%A5\%E9\%80\%89\%E8\%AE\%BA\%E6 \%96\%87/\%E4\%BA\%A7\%E4\%B8\%9A\%E7\%BB\%84\%E7\% BB\%87/Wang\%20Hao\%20CEApaper.pdf 